\title{
ORIGINAL RESEARCH \\ MR Imaging Assessment of Lumbar Intervertebral Disk Degeneration and Age-Related Changes: Apparent Diffusion Coefficient versus T2 Quantitation
}

\author{
G. Niu \\ J. Yang \\ R. Wang \\ BACKGROUND AND PURPOSE: T2 and ADC mappings are 2 quantitative MR imaging tools for assess- \\ ing IVDD. This study aimed to compare these 2 measures in detecting IVDD and its age-related \\ changes.
}

S. Dang

E.X. Wu

Y. Guo

\begin{abstract}
MATERIALS AND METHODS: Thirty-seven asymptomatic volunteers and 28 patients with back pain or sciatica were examined, and their lumbar disk T2 and ADC maps were quantified via sagittal imaging protocols at 1.5T. For all participants, the Pfirrmann system was used by 2 radiologists for grading disks. T2 and ADC values in the inner portion of disks were measured, and their variances in different grades were analyzed by 1-way ANOVA testing. The ability of T2 and ADC measures to differentiate IVDD grades was compared on the basis of their ROC curves. For asymptomatic subjects, the correlations between age and the $2 \mathrm{MR}$ imaging measures were assessed by the Pearson correlation test.

RESULTS: Both T2 and ADC values were found to decrease with the increasing Pfirrmann grades except T2 in grade V. Significant T2 differences were seen among grades I-IV, but not between grades IV and V. There were no significant ADC differences among grades I-III. Moreover, the areas under the ROC curves differed significantly $(0.95$ and 0.67 for $T 2$ and ADC, respectively). Linear regression analysis revealed that $\mathrm{T} 2$ yielded more significant correlation with age $(r=-0.77)$ than ADC did $(r=$ $-0.37)$.
\end{abstract}

CONCLUSIONS: T2 quantitation provides a more sensitive and robust approach for detecting and characterizing the early stage of IVDD and age-related disk changes.

ABBREVIATIONS: $A D C=$ apparent diffusion coefficient; ANOVA = analysis of variance; IVDD = intervertebral disk degeneration; $\mathrm{ROC}=$ receiver operating characteristic analysis; $\mathrm{T} 2 \mathrm{WI}=\mathrm{T} 2$ weighted imaging

lones VDD is a leading cause of lumbar spine-related low back pain. ${ }^{1}$ Several imaging techniques have been used to detect IVDD. Radiography and conventional CT are the 2 common

Received November 4, 2010; accepted after revision January 18, 2011.

From the Department of Diagnostic Radiology (G.N., J.Y., R.W.) and Molecular Imaging Center (Y.G.), First Hospital of Medical School, Xi'an Jiaotong University, Xi'an, China; Department of Epidemiology and Health Statistics (S.D.), Medical School of Xi'an Jiaotong University, Xi'an, Shannxi Province, China; and Laboratory of Biomedical Imaging and Signal Processing (E.X.W.), University of Hong Kong, Pokfulam, Hong Kong, SAR, China.

Dr Gang Niu and Professor Jian Yang contributed equally to this study. Dr Gang Niu made a substantial contribution to MR imaging data acquisition, literature research, statistical analysis, and manuscript drafting. Professor Jian Yang made a substantial contribution to the concept and design of the study, the analysis and interpretation of the results, and the writing of the article. Dr Rong Wang participated in the MR imaging data acquisition and imaging data analysis. Professor Shaonong Dang participated in the design of the study and statistical analysis. Professor Ed X. Wu participated in the design of the study, manuscript editing, and modifying language. Professor Youmin Guo participated in the design of study and guidance, editing, and organization of the article. All authors have approved the final submitted version.

This work was supported in part by the National Natural Foundation of China (grant 30970797 to Jian Yang) and the National Basic Research Program of China (973 Program, grant 2010CB7332603)

Please address correspondence to Jian Yang, MD, PhD, Department of Diagnostic Radiology, First Hospital of Medical School, Xi'an Jiaotong University, Xi'an, China; e-mail: cjr.yangjian@vip.163.com; or Youmin Guo, MD, PhD, Molecular Imaging Center, First Hospital of Medical School, Xi'an Jiaotong University, Xi'an, China; e-mail: cjr.guoyoumin@ vip.163.com

Indicates open access to non-subscribers at www.ajnr.org

http://dx.doi.org/10.3174/ajnr.A2556 imaging methods for evaluation of disk morphology and reactive bony changes. ${ }^{1,2}$ However, the biochemical events during early IVDD, including the breakdown of large aggregating proteoglycan gels and extracellular matrix degradation, occur before the morphologic changes and alterations in biomechanical properties. Therefore, the 2 aforementioned methods are generally unable to detect IVDD at an early stage. ${ }^{2}$

MR imaging can reflect both the macromolecular concentrations and the structural integrity in the intervertebral disk and has been considered the best noninvasive method of characterizing IVDD in humans and animal models in vivo., Conventional clinical MR imaging emphasizes the signal intensity and morphologic changes of intervertebral disks in T2WI. ${ }^{3}$ Investigators have reported several IVDD classification systems based on sagittal T2WI, ${ }^{5-7}$ among which the Pfirrmann scoring system is widely used as an accredited standard in both research and preliminary clinical applications. ${ }^{8-15}$ It is a subjective rating system and can provide a semiquantitative assessment of IVDD in vivo. ${ }^{8,12-14}$ For further quantitative evaluation of IVDD based on the biochemical changes in disks, several MR imaging techniques have been developed. They include dynamic contrast MR imaging, ${ }^{9}$ magnetization transfer imaging, ${ }^{16} \mathrm{~T} 1$ measurement, ${ }^{17} \mathrm{~T} 2$ measurement, ${ }^{10-14,17-21}$ T1 $\rho$ imaging, ${ }^{13,15,22}$ MR spectroscopy, ${ }^{2}$ and diffusionweighted imaging. ${ }^{8,19,23-25}$ These recently proposed tech- 
niques aim to quantitatively detect early degenerative changes in the matrix content of intervertebral disks.

The lumbar intervertebral disk is the largest avascular structure in a human body. Nutrition depends on the diffusion of fluid either from the vertebral bodies across cartilaginous endplate or through the annulus fibrosus from the surrounding capillaries. ${ }^{26}$ ADC, a quantitative parameter calculated from diffusion-weighted imaging, is sensitive in detecting the molecular diffusibility in tissue. Previous studies have demonstrated that decreased diffusion is associated with a reduction in nutrient supply in IVDD. ${ }^{27}$ ADC mapping could reflect the degenerative changes of disk matrix composition and integrity. ${ }^{23-25}$ Moreover, the signal intensity of T2WI in the disk was found to correlate with the ADC values. ${ }^{8}$

The quantitative T2 has been reported to reflect the molecular environment in the disk, which consists of water, proteins, fat, collagen, and other solutes. Increased disk water or glycosaminoglycan content, the primary component of proteoglycan, is associated with increased T2, whereas increased collagen content contributes to decreased T2. ${ }^{20}$ Numerous studies have revealed that degenerating intervertebral disks have shorter T2 values than normal disks ${ }^{11,12}$ and that T2 correlates with the Pfirrmann grades. ${ }^{14}$ Moreover, disk T2 varies with age, ${ }^{18}$ magnitude, or duration of compression ${ }^{21}$ and diurnal variation. ${ }^{19}$ Therefore, T2 may serve as a potential quantitative and sensitive biomarker for detecting the IVDD at an early stage.

At present, T2 and ADC are the 2 major MR imaging measures for the quantitative evaluation of IVDD. There has been no study so far to evaluate and compare their clinical performance in the assessment of intervertebral degenerative disk disease. The primary aim of the current study was to evaluate the correlation between the Pfirrmann grades and these $2 \mathrm{MR}$ imaging measures and to determine which technique is more sensitive in detecting the early stage of IVDD. The secondary aim was to evaluate how T2 and ADC vary with age in a welldefined asymptomatic population and to acquire further evidence on which measure is more suitable for detecting agerelated lumbar intervertebral disk degeneration. The results of this study would contribute to establishing the optimal clinical diagnosis paradigm in IVDD management.

\section{Materials and Methods}

\section{Subjects}

This prospective study was approved by the local institutional review board. All participants were informed of the aims and risks of MR imaging, and written consent was obtained before enrollment. They were consecutively recruited and studied from September 2009 to May 2010. The subjects were excluded if they had diabetes mellitus, major systemic disease, serious illness (eg, tumor, infection), back surgery, spinal fractures, or osteoporosis. They consisted of asymptomatic volunteers and patients with back pain and/or sciatica. The inclusion criteria of patients were the presence of significant back pain, which was defined as a pain in the low back for $>2$ weeks and severe enough to require physician consultation or treatment. ${ }^{28}$ Moreover, inclusion criteria of asymptomatic volunteers referenced a report by Pfirrmannet $\mathrm{al},{ }^{29}$ which observed the process of normal aging in the lumbar disks, as follows: 1) no back pain within the last 5 years, 2) never absent from work because of low back pain, and 3) no history of consulting a physician because of low back pain.

\section{MR Imaging}

Lumbar spine imaging was performed on a whole-body $1.5 \mathrm{~T}$ clinical scanner (Gyroscan, release 9.1.2; Philips Healthcare, Best, the Netherlands). All MR images in this study were obtained in the afternoon to minimize the diurnal variation of $\mathrm{ADC}$ and $\mathrm{T} 2$ values in the intervertebral disks. ${ }^{19}$

The conventional lumbar MR images were acquired with a 4-channel cervical-thoracic-lumbar spinal radio-frequency coil (Syn spine, Philips Healthcare) by T2WI using a fast spin-echo sequence in sagittal and axial orientations. The scanning parameters were as follows: $\mathrm{TR} / \mathrm{TE}=3500 / 120 \mathrm{~ms}$, thickness $=4.0 \mathrm{~mm}, \mathrm{FOV}=325 \mathrm{~mm}$, matrix $=512 \times 512$, number of signal-intensity acquisitions $=4$, turbo factor $=17$, number of sagittal sections $=11$, number of axial sections in each disk $=3$, and acquisition time $=4$ minutes 29 seconds. In addition, the sagittal T1-weighted imaging was performed by using a fast spin-echo sequence with $\mathrm{TR} / \mathrm{TE}=400 / 14 \mathrm{~ms}$, thickness $=$ $4.0 \mathrm{~mm}, \mathrm{FOV}=256 \mathrm{~mm}$, matrix $=512 \times 512$, number of signal intensity acquisitions $=2$, turbo factor $=3$, number of sections $=11$, and acquisition time $=2$ minutes 56 seconds. The sagittal T2WIs were used for the visual Pfirmann grading of IVDD.

The sagittal diffusion-weighted images of the lumbar spine were obtained by using a single-shot spin-echo echo-planar imaging sequence coil with the diffusion gradient applied in 3 orthogonal directions, respectively, from which the average ADC map was calculated. In theory, the optimal b-value should be in the range of the inverse of the mean ADC of the tissue examined $\left(1.99 \times 10^{-3} \mathrm{~mm}^{2} / \mathrm{s}\right.$ for disk $)$ to obtain the optimal signal intensity-to-noise ratio in the diffusion map. ${ }^{19}$ For this reason, a b-value of $500 \mathrm{~s} / \mathrm{mm}^{2}$ was used in this study. Other parameters were the following: TR/TE $=3000 / 81 \mathrm{~ms}$, thickness $=6.0 \mathrm{~mm}$, FOV $=250 \mathrm{~mm}$, matrix $=256 \times 256$, and number of signal intensity acquisitions $=8$. The total scanning time was 3 minutes 24 seconds for 3 sections passing through the middle of the lumbar spine.

T2 mapping was performed by a hybrid turbo sequence with 2 effective TEs per acquisition by using a body radio-frequency coil. This hybrid turbo (turbo-mixed) sequence consists of interleaved spin-echo and inversion-recovery acquisitions and has the ability to map the $\mathrm{T} 1$ and $\mathrm{T} 2$ values from a single measurement. ${ }^{30}$ We used the following parameters: TR/TE1/TE2 $=3500 / 5.88 / 100 \mathrm{~ms}$ for spinecho acquisitions, TR/TE1/TE2 $=2500 / 5.88 / 100 \mathrm{~ms}$ and $\mathrm{TI}=500 \mathrm{~ms}$ for the inversion-recovery acquisitions, turbo factor $=22$, FOV $=$ $250 \mathrm{~mm}$, section thickness $=8.0 \mathrm{~mm}$, matrix $=230 \times 256$, reconstruction matrix $=512 \times 512$, number of signal-intensity acquisitions $=2$, and anterior saturation bands. A single midline sagittal section was positioned parallel to each lumbar intervertebral disk from L1-2 to L5-S1. The scanning time was 2 minutes 16 seconds. T2 maps were computed on the basis of the least-squares fitting algorithm by using the software tools provided by the MR imaging system vendor.

\section{Pfirrmann Grading of Disks}

IVDD grading was performed in the sagittal T2WI by 2 experienced radiologists (with 17 and 13 years of experience, respectively, in MR imaging of the spine). They independently reviewed each intervertebral disk by the Pfirrmann criteria from L1-2 to L5-S1. Note that the grading system was primarily based on the changes of signal intensity 
Table 1: The modified Pfirrmann grading of disk degeneration

\begin{tabular}{lllll}
\hline & \multicolumn{1}{c}{ Structure } & $\begin{array}{c}\text { Distinction of Nucleus Pulposus } \\
\text { and Annulus Fibrosus }\end{array}$ & Signal Intensity & $\begin{array}{c}\text { Height of Intervertebral } \\
\text { Disk }\end{array}$ \\
\hline I & Homogeneous, bright white & Clear & Hyperintense & Normal \\
II & Inhomogeneous with or without horizontal bands & Clear & Hyperintense & Normal \\
III & Inhomogeneous, gray & Unclear & Intermediate & Normal-to-slight decrease \\
IV & Inhomogeneous, gray to black & Lost & Intermediate to hypointense & Normal-to-moderate decrease \\
V & Inhomogeneous, black & Lost & Hypointense & Collapsed disk space \\
\hline
\end{tabular}

\section{Region of interest setting}

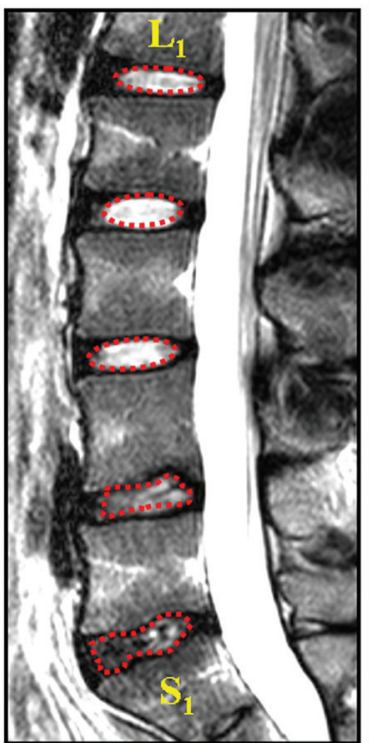

$\mathbf{T}_{2} \mathbf{W I}$

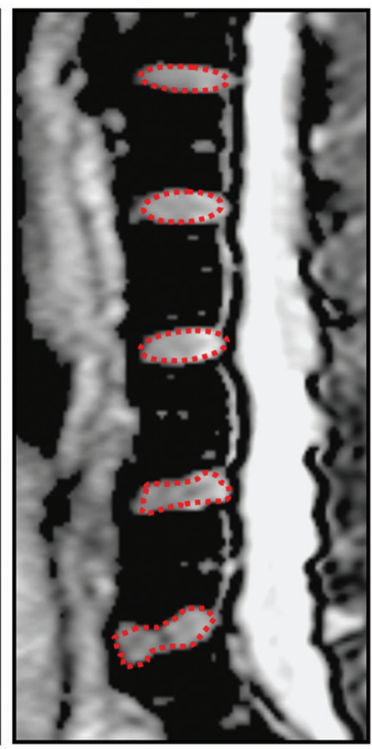

ADC Map

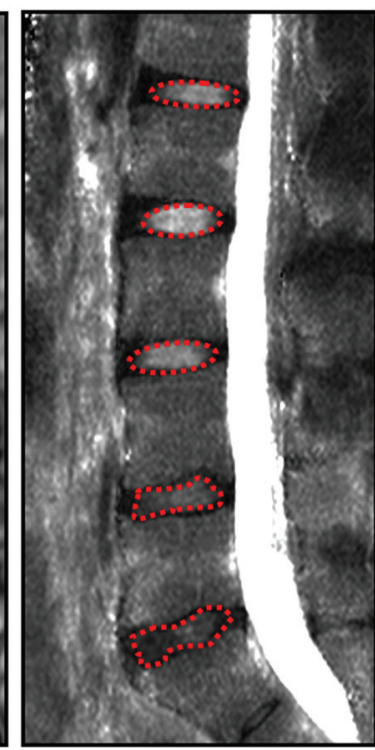

$\mathbf{T}_{2}$ Map

Fig 1. Illustration of regions of interest for T2 and ADC measurements in a 20-year-old man with low back pain. The elliptic regions of interest with red dotted lines are manually delineated in T2WI at the center of lumbar disks to cover the nucleus pulposus and inner annulus fibrosus from L1 to L4. The irregular regions of interest for L4-S1 were selected by the operator according to the expected location of inner portion of lumbar disks. Then these regions of interest in T2WI were copied to the ADC and T2 maps, and T2 and ADC values were measured.

in the nucleus, distinction between the nucleus and annulus, and disk height. ${ }^{2,7,31}$ However, it was not used as a criterion for grades I and II, in which the signal intensity of the intervertebral disk was generally isointense to that of CSF (Table 1). ${ }^{2,31}$

To estimate the intraobserver and interobserver reliability, the 2 radiologists, without knowledge of the findings on ADC and T2 maps, reviewed each case twice with a 3-week time lapse. Individual results from the 2 reviewers were tabulated and compared. If any disagreement occurred during the reviews on the Pfirrmann grade, the sagittal T2WI would be reviewed together by the 2 radiologists to reach a consensus.

\section{Region-of-Interest Setting}

All data were transferred to the imaging workstation (EasyVision, Philips Healthcare) for analysis. A radiologist with $>5$ years' experience in spine MR imaging delineated the region of interest in the disks twice within a 2 -week interval. For disks with Pfirrmann grades I and II, an elliptic region of interest, on the middle section of the sagittal T2WI, was manually drawn on the inner portion of each lumbar disk with areas of $60 \sim 80 \mathrm{~mm}^{2}$ to cover the nucleus pulposus and inner annulus fibrosus, which had high signal intensity on T2WI. ${ }^{3}$ For disks with Pfirrmann grades III, IV, and V, where the boundary between the inner portion and the outer annulus fibrosus was not clearly distinguishable, an irregular region of interest was delineated by the operator according to the expected location of the inner portion. ${ }^{14}$ These regions of interest on T2WI were copied to the $\mathrm{ADC}$ and $\mathrm{T} 2$ maps at same level (Fig 1), and T2 and ADC values were measured as mean $\pm \mathrm{SD}$.

\section{Statistical Analysis}

To evaluate the reliability of Pfirrmann grading, we tested intraobserver and interobserver agreements by using $\kappa$ statistics. The level of agreement was assessed by complying with the criteria of $\kappa$ values reported by Landis and $\mathrm{Koch}^{32}$ as follows: negative $\kappa$ value for poor agreement, $0.00-0.20$ for slight agreement, $0.21-0.40$ for fair agreement, $0.41-0.60$ for moderate agreement, $0.61-0.80$ for good agreement, and $>0.81$ for excellent agreement.

A paired 2-tailed Student $t$ test was used to compare the T2 and $\mathrm{ADC}$ values between the patient and volunteer groups. Moreover, the variations of $\mathrm{T} 2$ and ADC values with age were analyzed by the Pearson correlation in the asymptomatic group. The degree of correlation was assessed as follows: absolute correlation coefficient $(r): r>0.7$ as strong, $0.5<r \leq 0.7$ as moderate, and $r \leq 0.5$ as weak.

To determine the statistical differences of $\mathrm{T} 2$ and $\mathrm{ADC}$ values between the different Pfirrmann grades, 1-way ANOVA was used with the Student Newman-Keuls test. Moreover, ROC curves were plotted to test the sensitivity and specificity of T2 and ADC measures 


\begin{tabular}{|c|c|c|c|c|c|c|}
\hline \multirow[b]{2}{*}{ Observers } & \multicolumn{5}{|c|}{ Agreement (\%) } & \multirow[b]{2}{*}{$\begin{array}{c}\kappa \\
\text { Value }\end{array}$} \\
\hline & $\begin{array}{c}\text { Grade } \\
\text { I }\end{array}$ & $\begin{array}{c}\text { Grade } \\
\text { II }\end{array}$ & $\begin{array}{c}\text { Grade } \\
\text { III }\end{array}$ & $\begin{array}{c}\text { Grade } \\
\text { IV }\end{array}$ & $\begin{array}{c}\text { Grade } \\
\text { V }\end{array}$ & \\
\hline \multicolumn{7}{|l|}{ Intraobserver } \\
\hline $\mathrm{A} 1-\mathrm{A} 2$ & 76.7 & 89.0 & 83.1 & 71.6 & 87.5 & 0.795 \\
\hline B1-B2 & 90.7 & 91.2 & 79.8 & 51.0 & 87.5 & 0.822 \\
\hline \multicolumn{7}{|l|}{ Interobserver } \\
\hline $\mathrm{A} 1-\mathrm{B} 1$ & 72.1 & 83.8 & 75.3 & 51.0 & 87.5 & 0.675 \\
\hline A1-B2 & 74.4 & 89.7 & 75.3 & 61.2 & 87.5 & 0.730 \\
\hline A2-B1 & 86.0 & 86.8 & 80.9 & 53.1 & 87.5 & 0.722 \\
\hline A2-B2 & 90.7 & 94.1 & 83.1 & 63.3 & 87.5 & 0.812 \\
\hline
\end{tabular}

for assessing Pfirrmann grading. Areas under the ROC curve were calculated. The statistical difference between T2 and ADC measures for assessing Pfirrmann grading was determined by a $U$-test. All tests above were considered significant with $P<.05$. All statistical analyses were performed by using the Statistical Package for the Social Sciences for Windows, Version 13.0.0 (SPSS, Chicago, Illinois).

\section{Results}

According to the inclusion and exclusion criteria in this study, 37 asymptomatic volunteers ( 25 men and 12 women; mean age, 40 years; median age, 47 years; age range, $21-73$ years) and 28 symptomatic patients ( 10 men and 18 women; mean age, 49 years; median age, 40 years; age range, 20-74 years) were recruited.

\section{Pfirmann Grading}

A total of 325 intervertebral disks were categorized as Pfirrmann grades I $(n=43)$, II $(n=136)$, III $(n=89)$, IV $(n=$ $49)$, and V $(n=8)$. Except for 1 disk due to susceptibility artifacts, 324 disks were successfully measured for T2 values. Three hundred nineteen disks were measured for ADC values with confidence, and 6 disks were excluded because 4 disks at L4-L5 had susceptibility artifacts, whereas the other 2 disks at L5-S1 were grade V and too narrow to define regions of interest reliably.

Regarding the Pfirrmann grading reproducibility by the 2 readers, the intraobserver test yielded $\kappa$ values ranging from 0.795 to 0.822 , whereas the interobserver test produced $\kappa$ values of $0.675-0.812$ (Table 2). The main reason for disagreement in grading disks was the difficulty in distinguishing the inner portion and outer annulus fibrosus.

\section{T2 and ADC Values: Volunteers versus Patients}

The mean T2 value in the 139 disks from the patient group was $97 \pm 35 \mathrm{~ms}$. It was significantly less than that in the 185 disks from the volunteer group ( $115 \pm 42 \mathrm{~ms}, 34 \%$ reduction, $P<$ $.001)$. Similarly, the mean ADC value in the 134 disks from the patient group was significantly lower than that in the 185 disks from the volunteer group $\left(1.93 \pm 0.36 \times 10^{-3} \mathrm{~mm}^{2} / \mathrm{s}\right.$ versus $2.03 \pm 0.40 \times 10^{-3} \mathrm{~mm}^{2} / \mathrm{s}, 10 \%$ reduction, $\left.P<.001\right)$.

\section{Correlation with Age: T2 versus ADC}

A well-defined asymptomatic population of volunteers was recruited and divided into 5 groups according to age (Table 3 ). Both T2 and ADC values in their lumbar disks were found to correlate with age (Fig 2). Linear regression analysis revealed

\begin{tabular}{|c|c|c|c|c|}
\hline $\begin{array}{l}\text { Age Group } \\
\text { (yr) }\end{array}$ & No. & $\%$ & $\begin{array}{l}\text { T2 (ms) } \\
\text { (mean) }\end{array}$ & $\begin{array}{c}\mathrm{ADC}\left(\times 10^{-3} \mathrm{~mm}^{2} / \mathrm{s}\right) \\
(\text { mean })\end{array}$ \\
\hline $20-29$ & 8 & $21.6 \%$ & $164 \pm 31$ & $2.09 \pm 0.25$ \\
\hline 30-39 & 10 & $27.0 \%$ & $122 \pm 30$ & $2.07 \pm 0.24$ \\
\hline $40-49$ & 8 & $21.6 \%$ & $115 \pm 25$ & $2.25 \pm 0.22$ \\
\hline $50-59$ & 5 & $13.5 \%$ & $84 \pm 26$ & $1.96 \pm 0.34$ \\
\hline$>60$ & 6 & $16.2 \%$ & $65 \pm 16$ & $1.64 \pm 0.64$ \\
\hline Total/average & 37 & $100 \%$ & $115 \pm 42$ & $2.03 \pm 0.40$ \\
\hline
\end{tabular}

that T2 exhibited a more significant inverse correlation with age $(r=-0.77, P<.01)$ than $\operatorname{ADC}(r=-0.37, P<.01)$.

\section{Assessment of Pfirrmann Grading: T2 versus ADC}

A typical set of disks containing the Pfirrmann grades I, II, III, and IV are shown in Fig 1, with their T2 and ADC maps. For all disks from volunteers and patients, the mean and individual T2 and ADC values for each Pfirrmann grade are shown in Table 4 and Fig 3. Both T2 and ADC decreased with the increase of Pfirrmann grades except $\mathrm{T} 2$ in grade $\mathrm{V}$. As shown in Fig $3 A$, significant differences in T2 were observed between any 2 grades (ANOVA, $P<.05$ ) except between grades IV and $\mathrm{V}$ (ANOVA, $P>.05$ ). Figure $3 B$ shows the significant differences in ADC found between grades I and IV, grades II and IV, grades III and IV, and grades IV and V (ANOVA, $P<.05$ ). No significant differences in ADC were seen between grades I and II and II and III (ANOVA, $P>.05$ ). These results indicate that $\mathrm{T} 2$ is more sensitive than ADC in detecting the early stage of IVDD and that the ability exists for T2 and ADC maps to distinguish the different Pfirrmann grades with a significant difference. As shown in Fig 4, the areas under the ROC curves were $0.95(\mathrm{SE}=0.014)$ and $0.67(\mathrm{SE}=0.036)$ for $\mathrm{T} 2$ and $\mathrm{ADC}$, respectively. Their difference was statistically significant as determined by the $U$-test $(P<.05)$. This finding further suggests that $\mathrm{T} 2$ offers a more robust measure to differentiate IVDD stages than ADC.

\section{Discussion}

In this prospective study, $\mathrm{T} 2$ and $\mathrm{ADC}$ values in the inner portion of lumbar intervertebral disks were measured in symptomatic patients and asymptomatic volunteers and then compared against the Pfirrmann grades. Their negative correlations with disk-degeneration grades were demonstrated. More important, the results indicated that T2 was more sensitive than ADC in differentiating Pfirrmann grades I, II, and III. To the best of our knowledge, the present study is the first to use a well-defined asymptomatic population to examine the age-related T2 and ADC changes in the inner portion of intervertebral disks. Linear regression analysis of T2 versus age demonstrated a more significant inverse correlation than that of ADC versus age. These results indicate that compared with $\mathrm{ADC}, \mathrm{T} 2$ is a more sensitive and robust measure for quantitatively detecting early stage and age-related changes in IVDD.

In the present study, mean ADC values in the inner portion of disks were close to those reported in studies by Kealey et $\mathrm{al}^{24}$ and Niinimäki et al, ${ }^{8}$ who used the sagittal plane for diffusionweighted imaging and b-values of 400 and $500 \mathrm{~s} / \mathrm{mm}^{2}$, respectively. However, our measurement of mean ADC in normal disks was higher than those previously reported by Kurunlahti 

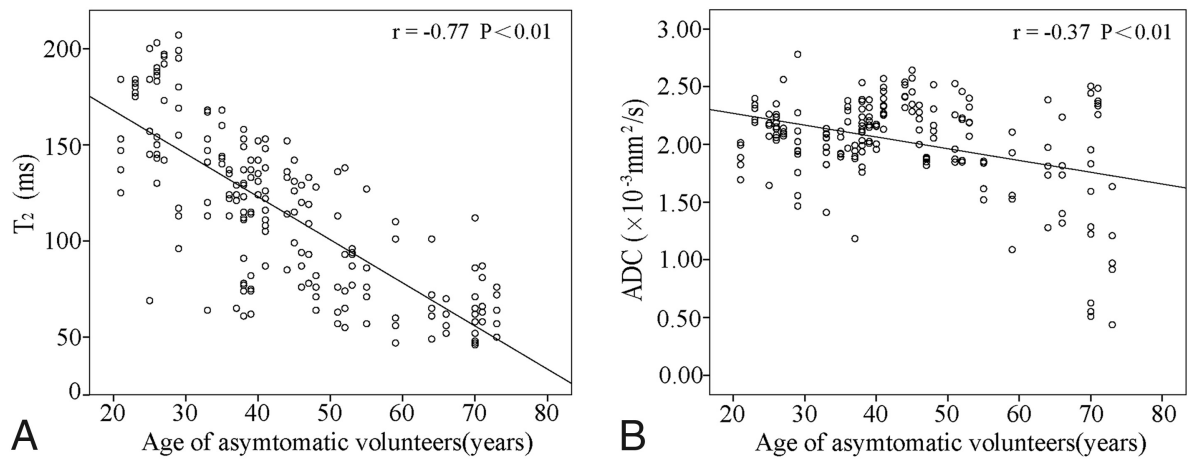

Fig 2. The correlations of the $T 2$ and $A D C$ measurements in lumbar disks with age in asymptomatic volunteers. The solid line represents the regression line.

\begin{tabular}{|c|c|c|c|c|}
\hline Grade & No. & $\begin{array}{l}\text { T2 (ms) } \\
\text { (mean) }\end{array}$ & No. & $\begin{array}{c}\mathrm{ADC}\left(\times 10^{-3} \mathrm{~mm}^{2} / \mathrm{s}\right) \\
(\text { mean })\end{array}$ \\
\hline I & 43 & $166 \pm 23$ & 43 & $2.16 \pm 0.19$ \\
\hline$\|$ & 135 & $128 \pm 22$ & 136 & $2.13 \pm 0.23$ \\
\hline III & 89 & $79 \pm 16$ & 89 & $1.96 \pm 0.32$ \\
\hline IV & 49 & $58 \pm 12$ & 45 & $1.58 \pm 0.48$ \\
\hline V & 8 & $59 \pm 10$ & 6 & $0.99 \pm 0.60$ \\
\hline Total & 324 & $107 \pm 40$ & 319 & $1.99 \pm 0.38$ \\
\hline
\end{tabular}

et $\mathrm{al}^{33}$ and Kerttula et al. ${ }^{34,35}$ In our study, mean ADC values in normal disks (grades I and II) ranged from $2.13 \pm 0.23 \times 10^{-3}$ $\mathrm{mm}^{2} / \mathrm{s}$ to $2.16 \pm 0.19 \times 10^{-3} \mathrm{~mm}^{2} / \mathrm{s}$; in these previous studies, these values ranged from $1.38 \times 10^{-3}$ to $1.60 \times 10^{-3} \mathrm{~mm}^{2} / \mathrm{s}$ in the $x, y$, and $z$ directions. This difference may arise from the varying partial volume effects because of their use of the axial plane for diffusion-weighted imaging. Moreover, ADC values decreased with the increase of the Pfirrmann grade and age in our study. These are in disagreement with the study by Niinimäki et al. ${ }^{8}$ In that study, only disk levels L3-L4, L4-L5, and L5-S1 were analyzed in middle-aged men; Pfirrmann grade I disks were not observed and grade II disks were, in a consensus reading, reclassified as grade III. $^{8}$ Therefore, the discrepancies may be attributed to the different study populations, different sampling of disk levels, and different grading criteria.

In previous studies, ${ }^{10,12,14}$ disk T2 was measured with a relatively long acquisition time by using a multiecho spinecho sequence, making its routine clinical use difficult for detection of early IVDD. In this study, T2 mapping with high spatial resolution $\left(0.49 \times 0.49 \mathrm{~mm}^{2}\right)$ and a short scanning time ( 2 minutes 16 seconds) was performed by using a hybrid turbo sequence with 2 effective TEs, which has been proved feasible and relatively accurate for T2 mapping in cartilage. ${ }^{30}$ Our preliminary evaluation of the specific T2 mapping sequence used in the current study indicated that its measurement of $\mathrm{T} 2$ in intervertebral disks is within $\pm 2.5 \%$ of that measured by a 22-echo spin-echo sequence (data not shown). Moreover, the mean T2 values for Pfirrmann grades II-V in this study $(128 \pm 22 \mathrm{~ms}$ to $58 \pm 12 \mathrm{~ms})$ were close to those of a recently published study $(108 \pm 23 \mathrm{~ms}$ to $53 \pm 11 \mathrm{~ms}),{ }^{14}$ further corroborating the applicability of this fast sequence for T2 mapping in IVDD. In another recent study, ${ }^{13} \mathrm{~T} 2$ in the disks of various Pfirrmann grades (grades I-IV, $92.3 \pm 27.2 \mathrm{~ms}$ to $37 \pm 0 \mathrm{~ms}$ ) was substantially lower than that in the current study, most likely because of the $3 \mathrm{~T}$ field used and the different composition of subjects examined. The magic angle effect is known to increase regional $\mathrm{T} 2$ values when collagen fibers are oriented $54.7^{\circ}$ relative to the orientation of the static magnetic field (B0). ${ }^{36}$ This effect can be present inevitably in the annulus fibrosus, especially near its surface. ${ }^{30}$ Given this scenario, the regions of interest in the present study only circumvented the nucleus pulposus and inner annulus fibrosus.

In the present study, T2 values were found to decrease with the extent of disk degeneration except in grade V (Table 4); actually there was no significant difference between grades IV $(58 \pm 12 \mathrm{~ms})$ and $\mathrm{V}(59 \pm 10 \mathrm{~ms})$. The findings of our study were mainly consistent with those of several previous studies. ${ }^{13,14}$ Moreover, the age-related reduction in $\mathrm{T} 2$ is associated with decreasing glycosaminoglycan and water content. ${ }^{37}$ Therefore, the inverse correlation between age and T2 has been observed in the current study and several previous studies. ${ }^{10,14}$ Because it is difficult to distinguish a painful degenerated (ie, pathologic) disk from one with age-related physiologic changes, a well-defined asymptomatic population was recruited for the current study in examining the effect of aging on T2. The population was different from that in a previous study by Marinelli et al, ${ }^{14}$ which observed the inverse correlation between age and T2 only for grade II disks in 20 patients with back pain or radiculopathy. Although asymptomatic volunteers were recruited and analyzed by Watanabe et al, ${ }^{10}$ the age distribution in that study had a narrow range of 20-44 years (mean age, $31.8 \pm 8.1$ years) compared with that in our study.

The principal finding from the current study is that T2 and ADC measures differ substantially in their respective ability to distinguish Pfirrmann grades, as manifested by the significantly different areas under the ROC curves (Fig 4). Furthermore, the T2 measure is more sensitive in differentiating the early stages of IVDD between grades I and III (Fig 3). This finding may be attributed to the different biophysical properties probed by $2 \mathrm{MR}$ imaging measures during IVDD. The T2 value in the intervertebral disk is known to be sensitive to water content and the composition of the collagen network structure. It is influenced by both rotational and translational motion by dipole-dipole interaction of water molecules in the collagen matrix. ${ }^{11-13,20}$ On the other hand, ADC is a parameter that is sensitive to both restricted diffusion of bound water in tissues and free water diffusion. Diffusion MR imaging merely reflects the translational motion of protons at the microscopic level. ${ }^{35}$ Thus, ADC may not perform better than T2 in evaluating water and collagen content, though it allows an indirect 

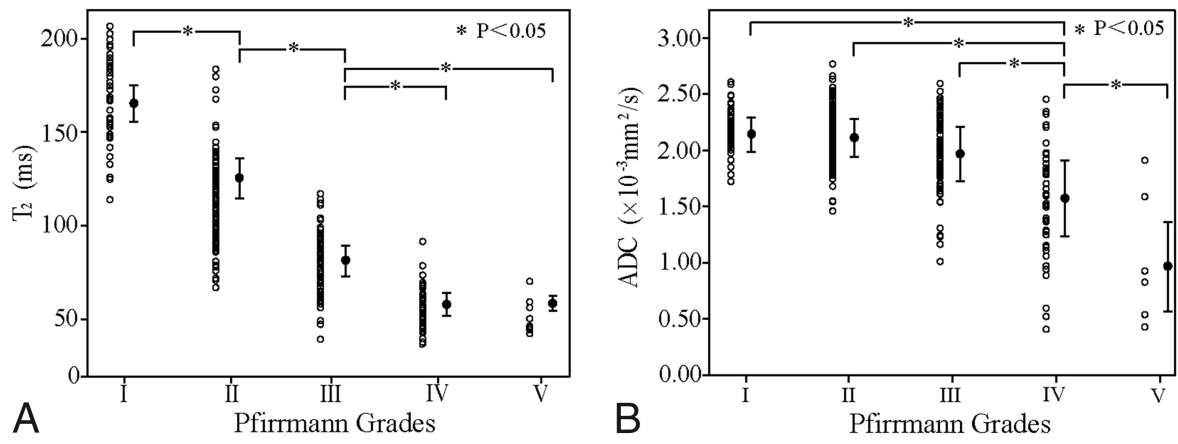

Fig 3. T2 $(A)$ and $A D C(B)$ measurements versus Pfirrmann grading

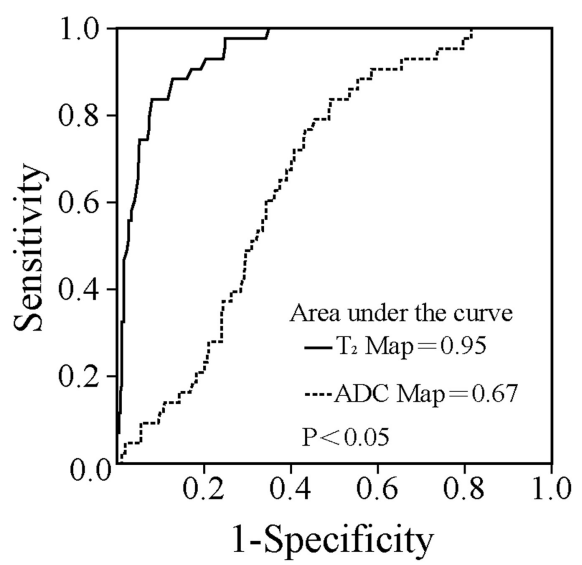

Fig 4. ROC curves of $T 2$ and $A D C$ in assessing the Pfirrmann grading. Note that the areas under the curves are computed. Their difference is statistically significant $(U$-test, $P<.05)$.

evaluation of disk-matrix composition and integrity. In a previous study, T2 was found to decrease dramatically in the nucleus pulposus from morning to evening, while no significant $\mathrm{ADC}$ change was detected, suggesting that $\mathrm{ADC}$ is a relatively insensitive measure for characterizing subtle changes of water and collagen content in the nucleus pulposus. ${ }^{19}$ Several studies have shown that the age-related IVDD can be detected by T2 changes, and age-related reduction in T2 is associated with decreasing glycosaminoglycan and water content. ${ }^{20,37,38}$ Using a well-defined asymptomatic cohort, the current study demonstrated a strong negative correlation between T2 and age $(r=-0.77)$ in contrast to the weak negative correlation between the ADC value and age $(r=-0.37)$, which clearly indicates the superior sensitivity of T2 in detecting intervertebral disk aging.

The current study had several limitations. First, a selection bias might exist in the enrollment of participants in all clinical prospective studies, which cannot be fully removed. The following measures were taken to minimize the effect of such selection bias in this study: First, we established the same exclusion criteria for all participants in the asymptomatic and symptomatic groups. Such exclusions included diseases such as diabetes mellitus, major systemic disease, tumor, infection, back surgery, spinal fractures, and osteoporosis, in which back pain or/and sciatica is one of the complications and the intervertebral disk is prone to degeneration. ${ }^{39-41}$ Moreover, the well-documented inclusion criteria were respectively applied for both asymptomatic volunteers and patients with back pain or/and sciatica. Second, the equivalence of age ranges and the

balanced age distribution were achieved in the asymptomatic and symptomatic groups. Third, because a study with a low level of participation is more vulnerable to subject-selection bias, ${ }^{42}$ a face-to-face interview was performed in the current study by a trained radiologist to minimize the drop-out rates $(<7 \%)$ in this study.

One major limitation in the current study was the lack of a criterion standard for staging IVDD. The ideal standard should assess intervertebral disks by biochemical and histologic methods, which was not feasible in this clinical and in vivo study. Up to now, several methods have been devised to stage disk degeneration. ${ }^{5,7,10,43}$ The Pfirrmann grading system, as a noninvasive, simple, and convenient MR imaging method, can provide a morphologic and semiquantitative evaluation of IVDD in vivo, and it exhibits good intrareader and inter-reader reliability. ${ }^{7-15,22}$ In the current study, the interobserver and intraobserver reliability was good or excellent ( $\kappa$ value ranges of $0.675-0.812$ and $0.795-0.822$, respectively). In the research field, this staging system has been widely accepted as the control criterion for assessing the degree of accuracy by several quantitative methods in the detection of IVDD, such as T2 and ADC measurement. ${ }^{8,10,12-14}$ Nevertheless, this grading method is a subjective rating system and may introduce an inevitable bias.

Another limitation was the manual delineation of the regions of interest to cover the inner portion of intervertebral disks. This could have introduced subjectivity and bias, especially when the central region was poorly differentiated from the outer annulus fibrosus in disks of Pfirrmann grades III-V.

Last, the T2 and ADC values used to differentiate the Pfirrmann grades and the age ranges are important in quantitative assessment of degeneration and age-related changes in the intervertebral disks, which can play a value role in clinical practice. However, the sample size in the current study, especially after further grade or age classification, was not adequate for computing the positive and negative predictive values of T2 and ADC for different Pfirrmann grades and age ranges. Multicenter studies remain to be conducted with larger sample sizes, more rigorous designs, and evidence-based reviews.

\section{Conclusions}

Our results demonstrated that $\mathrm{T} 2$ and $\mathrm{ADC}$ values in lumbar intervertebral disks negatively correlated with the Pfirrmann grades in IVDD and age-related disk degeneration. More important, $\mathrm{T} 2$ provides a more sensitive and robust MR imaging 
measure for quantitatively detecting the early stage and agingrelated changes in IVDD.

\section{References}

1. Luoma $\mathrm{K}$, Riihimaki $\mathrm{H}$, Luukkonen $\mathrm{R}$, et al. Low back pain in relation to lumbar disc degeneration. Spine (Phila Pa 1976) 2000;25:487-92

2. Zuo J, Saadat E, Romero A, et al. Assessment of intervertebral disc degeneration with magnetic resonance single-voxel spectroscopy. Magn Reson Med 2009;62:1140-46

3. Modic MT, Ross JS. Lumbar degenerative disk disease. Radiology 2007;245:43-61

4. Waris E, Eskelin M, Hermunen $\mathrm{H}$, et al. Disc degeneration in low back pain: a 17-year follow-up study using magnetic resonance imaging. Spine (Phila $\mathrm{Pa}$ 1976) 2007;32:681-84

5. Griffith JF, Wang YX, Antonio GE, et al. Modified Pfirrmann grading system for lumbar intervertebral disc degeneration. Spine (Phila Pa 1976) 2007;32: E708-12

6. Schneiderman G, Flannigan B, Kingston S, et al. Magnetic resonance imaging in the diagnosis of disc degeneration: correlation with discography. Spine (Phila Pa 1976) 1987;12:276-81

7. Pfirrmann CW, Metzdorf A, Zanetti M, et al. Magnetic resonance classification of lumbar intervertebral disc degeneration. Spine (Phila Pa 1976) 2001;26: $1873-78$

8. Niinimäki J, Korkiakoski A, Ojala O, et al. Association between visual degeneration of intervertebral discs and the apparent diffusion coefficient. Magn Reson Imaging 2009;27:641-47

9. Rajasekaran S, Babu JN, Arun R, et al. ISSLS prize winner: a study of diffusion in human lumbar discs-a serial magnetic resonance imaging study documenting the influence of the endplate on diffusion in normal and degenerate discs. Spine (Phila Pa 1976) 2004;29:2654-67

10. Watanabe A, Benneker LM, Boesch C, et al. Classification of intervertebral disk degeneration with axial T2 mapping. AJR Am J Roentgenol 2007;189:936-42

11. Perry J, Haughton V, Anderson PA, et al. The value of T2 relaxation times to characterize lumbar intervertebral disks: preliminary results. AJNR Am J Neuroradiol 2006;27:337-42

12. Trattnig S, Stelzeneder D, Goed S, et al. Lumbar intervertebral disc abnormalities: comparison of quantitative T2 mapping with conventional MR at 3.0 T. Eur Radiol 2010;20:2715-22

13. Blumenkrantz G, Zuo J, Li X, et al. In vivo 3.0-Tesla magnetic resonance T1rho and $\mathrm{T} 2$ relaxation mapping in subjects with intervertebral disc degeneration and clinical symptoms. Magn Reson Med 2010;63:1193-200

14. Marinelli NL, Haughton VM, Anderson PA. T2 relaxation times correlated with stage of lumbar intervertebral disk degeneration and patient age. AJNR Am J Neuroradiol 2010;31:1278-82

15. Nguyen AM, Johannessen W, Yoder JH, et al. Noninvasive quantification of human nucleus pulposus pressure with use of T1rho-weighted magnetic resonance imaging. J Bone Joint Surg Am 2008;90:796-802

16. Paajanen H, Komu M, Lehto I, et al. Magnetization transfer imaging of lumbar disc degeneration: correlation of relaxation parameters with biochemistry. Spine (Phila Pa 1976) 1994;19:2833-37

17. Chatani K, Kusaka Y, Mifune T, et al. Topographic differences of 1 H-NMR relaxation times $(\mathrm{T} 1, \mathrm{~T} 2)$ in the normal intervertebral disc and its relationship to water content. Spine (Phila Pa 1976) 1993;18:2271-75

18. Krueger EC, Perry JO, $\mathrm{Wu} \mathrm{Y}$, et al. Changes in $\mathrm{T} 2$ relaxation times associated with maturation of the human intervertebral disk. AJNR Am J Neuroradiol 2007;28:1237-41

19. Ludescher B, Effelsberg J, Martirosian P, et al. T2- and diffusion-maps reveal diurnal changes of intervertebral disc composition: an in vivo MRI study at 1.5 Tesla. J Magn Reson Imaging 2008;28:252-57

20. Marinelli NL, Haughton VM, Munoz A, et al. T2 relaxation times of intervertebral disc tissue correlated with water content and proteoglycan content. Spine (Phila Pa 1976) 2009;34:520-24

21. Drew SC, Silva P, Crozier S, et al. A diffusion and T2 relaxation MRI study of the ovine lumbar intervertebral disc under compression in vitro. Phys Med Biol 2004;49:3585-92

22. Johannessen W, Auerbach JD, Wheaton AJ, et al. Assessment of human disc degeneration and proteoglycan content using T1rho-weighted magnetic resonance imaging. Spine (Phila Pa 1976) 2006;31:1253-57

23. Antoniou J, Demers CN, Beaudoin G, et al. Apparent diffusion coefficient of intervertebral discs related to matrix composition and integrity. Magn Reson Imaging 2004;22:963-72

24. Kealey SM, Aho T, Delong D, et al. Assessment of apparent diffusion coefficient in normal and degenerated intervertebral lumbar disks: initial experience. Radiology 2005;235:569-74

25. Antoniou J, Mwale F, Demers CN, et al. Quantitative magnetic resonance imaging of enzymatically induced degradation of the nucleus pulposus of intervertebral discs. Spine (Phila Pa 1976) 2006;31:1547-54

26. Urban JP, Holm S, Maroudas A, et al. Nutrition of the intervertebral disc: effect of fluid flow on solute transport. Clin Orthop Relat Res 1982:296-302

27. Nguyen-minh C, Riley L 3rd, Ho KC, et al. Effect of degeneration of the intervertebral disk on the process of diffusion. AJNR Am J Neuroradiol 1997; 18:435-42

28. Cheung KM, Karppinen J, Chan D, et al. Prevalence and pattern of lumbar magnetic resonance imaging changes in a population study of one thousand forty-three individuals. Spine (Phila Pa 1976) 2009;34:934-40

29. Pfirrmann CW, Metzdorf A, Elfering A, et al. Effect of aging and degeneration on disc volume and shape: a quantitative study in asymptomatic volunteers. J Orthop Res 2006;24:1086-94

30. Van Breuseghem I, Bosmans HT, Elst LV, et al. T2 mapping of human femorotibial cartilage with turbo mixed MR imaging at $1.5 \mathrm{~T}$ : feasibility. Radiology 2004;233:609-14

31. Takatalo J, Karppinen J, Niinimaki J, et al. Prevalence of degenerative imaging findings in lumbar magnetic resonance imaging among young adults. Spine (Phila Pa 1976) 2009;34:1716-21

32. Landis JR, Koch GG. The measurement of observer agreement for categorical data. Biometrics 1977;33:159-74

33. Kurunlahti M, Kerttula L, Jauhiainen J, et al. Correlation of diffusion in lumbar intervertebral disks with occlusion of lumbar arteries: a study in adult volunteers. Radiology 2001;221:779-86

34. Kerttula LI, Jauhiainen JP, Tervonen O, et al. Apparent diffusion coefficient in thoracolumbar intervertebral discs of healthy young volunteers. J Magn Reson Imaging 2000;12:255-60

35. Kerttula L, Kurunlahti M, Jauhiainen J, et al. Apparent diffusion coefficients and $\mathrm{T} 2$ relaxation time measurements to evaluate disc degeneration: a quantitative MR study of young patients with previous vertebral fracture. Acta Radiol 2001;42:585-91

36. Xia Y. Magic-angle effect in magnetic resonance imaging of articular cartilage: a review. Invest Radiol 2000;35:602-21

37. Boos $\mathrm{N}$, Weissbach S, Rohrbach $\mathrm{H}$, et al. Classification of age-related changes in lumbar intervertebral discs: 2002 Volvo Award in basic science. Spine (Phila Pa 1976) 2002;27:2631-44

38. Leung VY, Hung SC, Li LC, et al. Age-related degeneration of lumbar intervertebral discs in rabbits revealed by deuterium oxide-assisted MRI. Osteoarthritis Cartilage 2008;16:1312-18

39. Robinson D, Mirovsky Y, Halperin N, et al. Changes in proteoglycans of intervertebral disc in diabetic patients: a possible cause of increased back pain. Spine (Phila Pa 1976) 1998;23:849-55, discussion 856

40. Lotan R, Oron A, Anekstein Y, et al. Lumbar stenosis and systemic diseases: is there any relevance? J Spinal Disord Tech 2008;21:247-51

41. Sierra-Jimenez G, Sanchez-Ortiz A, Aceves-Avila FJ, et al. Tendinous and ligamentous derangements in systemic lupus erythematosus. I Rheumatol 2008;35:2187-91

42. Bornehag CG, Sundell J, Sigsgaard T, et al. Potential self-selection bias in a nested case-control study on indoor environmental factors and their association with asthma and allergic symptoms among pre-school children. Scand J Public Health 2006;34:534-43

43. Thompson JP, Pearce RH, Schechter MT, et al. Preliminary evaluation of a scheme for grading the gross morphology of the human intervertebral disc. Spine (Phila Pa 1976) 1990;15:411-15 